\title{
Numerical treatment of coupled system of fractional order partial differential equations
}

\author{
Amjad Alia , Kamal Shahn ${ }^{\mathrm{a}}$, Yongjin Lib,*, Rahmat Ali Khan ${ }^{\mathrm{a}}$ \\ a Department of Mathematics, University of Malakand, Chakadara Dir(L), Khyber Pakhtunkhwa, Pakistan. \\ ${ }^{b}$ Department of Mathematics, Sun Yat-sen University, Guangzhou, P. R. China.
}

\begin{abstract}
This manuscript is devoted to the numerical solutions of coupled system of fractional partial differential equations (FPDEs). Using Legendre polynomials for two variables, we developed some operational matrices. Based on these matrices the considered coupled system is converted to some algebraic equations which can be easily solved for the unknown coefficient matrices needed in the approximate solutions of $u(x, t), v(x, t)$. The established technique is then applied to some numerical examples and the results are compared with some known wavelet methods, which demonstrate that our proposed method provides excellent solutions as compared to the other numerical methods.
\end{abstract}

Keywords: Shift Legendre polynomials, coupled system of partial differential equations, operational matrix, algebraic equation, numerical approximation.

2010 MSC: 37A25, 26A33, 26A42.

(C)2019 All rights reserved.

\section{Introduction}

In last few decades, the area devoted to the existence theory and numerical solutions of fractional ordinary differential equations (FODEs) and fractional partial differential equations (FPDEs) has gained the considerable attention of researchers $[9,18]$. This is because of its frequent use in all disciplines of science and engineering [3]. Many important phenomena in fluid flow, fluid dynamic like traffic model, electromagnetic, solid mechanics, statistical mechanics, colored noise, polarization, electrochemical processes, diffusion, modeling of frequency dependent damping behavior of viscoelastic flow, economics, and bioengineering are well described by partial differential equations and integro differential equations of non-integer order, see, for example $[1,2,12-15,19,21,22]$ and the references therein. Keeping in view, that in most cases fractional differential equations are very complicated to be solved exactly and although if an exact solution is obtained, that required very complicated calculations. Therefore, a lot of attention has been paid to the numerical solutions of fractional ordinary and partial differential equations. Several

\footnotetext{
*Corresponding author

Email addresses: amjadalimna@yahoo.com (Amjad Ali), kamalshah408@gmail.com (Kamal Shah), stslyj@mail.sysu.edu.cn (Yongjin Li), rahmat_alipk@yahoo.com (Rahmat Ali Khan)
}

doi: $10.22436 /$ jmcs.019.02.02

Received: 2017-09-19 Revised: 2019-03-31 Accepted: 2019-04-06 
efficient and powerful techniques are available dealing numerical approximations of FODEs and FPDEs. Some of the most commonly used methods are variational iteration method (VIM) [23], generalized differential transform method (GTM) [17], Adomain decomposition method (ADM) [7], He's variation iteration method (HVIM) [4], Chebyshev wavelets [11], Homotopy perturbation method [10], and Legendre polynomials approximation method [8].

Recently, numerical schemes based on operational matrices have gained the considerable attention of researches, due to their efficient and reliable numerical solutions to both linear and non linear partial differential equations of classical as well as fractional order. In this paper, we are determined to pull out the application of Shift Legendre polynomial to obtained the numerical solutions of coupled system of partial fractional differential equations given as

$$
\begin{aligned}
& { }^{c} D_{t}^{p} u(t, x)+{ }^{c} D_{x}^{q} v(t, x)=f(t, x), t, x \in[0,1], \\
& { }^{c} D_{t}^{q} v(t, x)+{ }^{c} D_{x}^{q} u(t, x)=g(t, x), t, x \in[0,1],
\end{aligned}
$$

subject to the initial conditions

$$
u(0, x)=\phi(x), v(0, x)=\theta(x)
$$

where ${ }^{c} D_{t}^{p}$ and ${ }^{c} D_{x}^{p}$ represent the Caputo fractional derivatives of order $p$, such that $0<p \leqslant 1$ w.r.t " $t$ " and " $x$ ", respectively. By developing operational matrices of fractional order integration and differentiation using Shifted Legendre polynomials, we convert the proposed system (1.1) to a system of algebraic equations. Solving established system of algebraic equations for unknown coefficient matrices, using them, we obtained the deserted solutions. In wide-range, algebraic equations of bulky system may lead to massive computational and needs large storage capacity. However, the techniques used in this paper are very straightforward and decrease the computational complexity. It is creditable to point out that the rooted technique in operational matrix of an orthogonal functions for solving FPDEs is computer oriented [8].

The rest of the paper is organized as follows. In Section 2, we presents some necessary definitions and preliminaries of fractional calculus and Legendre polynomial. In Section 3, we develops the operational matrices of fractional order derivatives and integration. In Section 4, we solve the coupled system of partial fractional differential equation. In Section 5, we illustrate the proposed method by some number of examples.

\section{Preliminaries}

The concerned section, is committed to the basic results, concepts and definitions of fractional calculus. Fractional calculus is generalization of classical calculus, which combines and generalizes the notations of integer order derivatives and n-fold integrals. There are numerous definitions of fractional order derivatives and integrals, such as Caputo, Riemann- Liouville and Grunwald-Letnikov's. Among these notation, we prefer Caputo type fractional order derivative and Riemann- Liouville type fractional integral through out this paper. The definitions of the needed materials are presented in the following sequel.

Definition 2.1 ([18]). The Riemann-Liouville integral of arbitrary order $p(p \geqslant 0)$ of a function $u(t)$ is defined by

$$
\mathcal{J}^{p} u(t)=\frac{1}{\Gamma(p)} \int_{0}^{t}(t-\tau)^{p-1} u(\tau) d \tau,
$$

where $\Gamma(p)=\int_{0}^{\infty} t^{p-1} e^{-t} d t$ is the gamma function and provided that the integral on right hand side converges pointwise on $(0, \infty)$. The Riemann-Liouville integral satisfies the following relations

(1) $\mathcal{J}^{p} \mathcal{J} q \mathfrak{u}(t)=\mathcal{J}^{q} \mathcal{J}^{p} \mathfrak{u}(t)$;

(2) $\mathcal{J}^{p} \mathcal{J} q u(t)=\mathcal{J}^{p}+q \mathfrak{u}(t)$; 
(3) $\mathcal{J p}^{\mathrm{p}} \mathrm{t}^{\mathrm{q}}=\frac{\Gamma(\mathrm{q}+1)}{\Gamma(\mathrm{p}+\mathrm{q}+1)} \mathrm{t}^{\mathrm{p}+\mathrm{q}}$.

Definition $2.2([18])$. For a given function $u(t)$, the Caputo fractional order derivative of order $p$ is defined as

$$
{ }^{c} \mathcal{D}^{p} u(t)=\frac{1}{\Gamma(n-p)} \int_{0}^{t} \frac{u^{(n)}(t)}{(t-\tau)^{p+1-n}} d \tau, n-1 \leqslant p<n, n=[p]+1,
$$

such that the right side is pointwise defined on $(0, \infty)$. Further, the operator ${ }^{c} \mathcal{D}$ satisfies the following properties in particular for any constant $C$,

$$
{ }^{c} \mathcal{D}^{p} c=0, \quad{ }^{c} \mathcal{D}^{p} t^{j}=\left\{\begin{array}{l}
0, j \in N, j<[p], \\
\frac{\Gamma(1+j)}{\Gamma(1+j-p)} t^{j-p} .
\end{array}\right.
$$

Some of the important results which are helpful in the rest of the paper are as follows.

Theorem 2.3 ([18]). For any function $\mathrm{u}(\mathrm{t})$, the following results hold.

(a) ${ }^{c} \mathcal{D}^{p} \mathcal{J p}^{p} u(t)=u(t)$;

(b) ${ }^{c} \mathcal{J}^{p} \mathcal{D}^{\mathfrak{p}} \mathfrak{u}(\mathrm{t})=\mathfrak{u}(\mathrm{t})-\sum_{j=0}^{n-1} u^{(j)} \frac{t^{j}}{\Gamma(j+1)}$;

(b) ${ }^{c} \mathcal{D}^{p}(\lambda u(t)+\mu v(t))=\lambda^{c} \mathcal{D}^{p} u(t)+\mu^{c} \mathcal{D}^{p} v(t)$.

\subsection{Shifted Legendre polynomials and their properties}

The Legendre basis polynomials defined on $[-1,1]$ are given by (for details see [20])

$$
W_{j+1}(z)=\frac{(2 j+1)}{(j+1)} z W_{j}(z)-\frac{j}{(j+1)} W_{j-1}(z), \quad j=1,2,3, \ldots,
$$

where $W_{0}(z)=1$ and $W_{1}(z)=2 z-1$. By taking the transformation $t=\frac{z+1}{2}$, which transforms the $[-1,1]$ to $[0,1]$, the shifted Legendre polynomial of degree $j$ is

$$
L_{j}(t)=\sum_{k=0}^{j}(-1)^{j+k} \frac{(j+k) ! t^{k}}{(j-k) !(k !)^{2}},
$$

where

$$
\mathrm{L}_{\mathfrak{j}}(0)=(-1)^{\mathfrak{j}} \text { and } \mathrm{L}_{\mathfrak{j}}(1)=1 \text {. }
$$

The orthogonality conditions is

$$
\int_{0}^{1} L_{j}(t) L_{k}(t) d t= \begin{cases}0, & j \neq k \\ \frac{1}{2 j+1}, & j=k\end{cases}
$$

Thus a function $u(t) \in \mathrm{L}^{2}(0,1)$ may be approximated in-term of shifted Legendre polynomials as

$$
u(t) \approx \sum_{j=0}^{m} E_{j} L_{j}(t),
$$

where the coefficients $E_{j}$ are given by

$$
E_{j}=\left\langle u(t), L_{j}(t)\right\rangle=(2 j+1) \int_{0}^{1} u(t) L_{j}(t) d t, j=1,2,3 \ldots
$$

In vector notation, we have

$$
u(t)=\mathbb{K}_{M}^{\top} \hat{S}_{M}
$$


where $\hat{S}$ is $M$ term vector function, $\mathbb{K}$ is coefficient vector, and $M=m+1$. Legendre polynomial in two variable of order $M=m+1$ as a product of two Legendre polynomials can be defined as

$$
L_{n}(t, x)=L_{i}(t) L_{j}(x), \quad n=M i+j+1, i, j=0,1,2,3, \ldots, m .
$$

The orthogonality condition for $u_{n}(t)$ can be written as

$$
\int_{0}^{1} \int_{0}^{1} L_{i}(t) L_{j}(x) L_{k}(t) L_{l}(x) d t d x= \begin{cases}0, & \text { if } i \neq k, j \neq l \\ \frac{1}{(2 i+1)(2 j+1)}, & \text { if } i=k, j=l\end{cases}
$$

Similarly, for the function $u(t, x) \in \mathrm{L}^{2}([0,1] \times[0,1])$, we get its approximation in term of Legendre polynomials as

$$
u(t, x) \approx \sum_{i=0}^{m} \sum_{j=0}^{m} E_{i j} L_{i}(t) L_{j}(x),
$$

where $E_{i j}$ can be written in a form of

$$
E_{i j}=(2 i+1)(2 j+1) \int_{0}^{1} \int_{0}^{1} u(t, x) L_{i}(t, x) L_{j}(t, x) d t d x .
$$

We represent the notation $E_{n}=E_{i j}$, where $n=M i+j+1$, then (2.2) implies

$$
u(t, x) \approx \sum_{n=1}^{M^{2}} E_{n} L_{n}(t, x)=\mathbb{K}_{M^{2}}^{\top} \Psi_{M^{2}}(t, x),
$$

where $\mathbb{K}_{M^{2}}$ is $M^{2} \times 1$ coefficient row vector and $\Psi_{M^{2}}(t, x)$ is $M^{2} \times 1$ column vector of functions provided as

$$
\Psi_{M^{2}(t, x)}=\left(\begin{array}{llllllll}
\psi_{11}(x, y) & \cdots & \psi_{1 M}(t, x) & \psi_{21}(t, x) & \cdots & \psi_{2 M}(t, x) & \cdots & \psi_{M M}(t, x)
\end{array}\right)^{\top},
$$

where $\psi_{j+1, k+1}(t, x)=\left(L_{j}(t)\right)\left(L_{k}(x)\right), j, k=0,1,2, \ldots, n$.

Theorem $2.4([5,6,22])$. If $\mathfrak{u}(t, x)$ is a continuous function defined over a region $[0,1] \times[0,1]$ has bounded mixed fourth order partial derivative $\frac{\partial^{2}}{\partial \mathrm{t}^{2} \partial \mathrm{x}^{2}} \mathrm{u}(\mathrm{t}, \mathrm{x})$, then the Legendre expansion of the function converges uniformly to the function. Moreover the error of the approximation for sufficiently smooth function $\mathrm{u}(\mathrm{t}, \mathrm{x})$ over the region $[0,1] \times[0,1]$ is given by

$$
\begin{aligned}
\left\|u(t, x)-S_{M}(t, x)\right\|_{2} \leqslant & {\left[\frac{1}{4} \max _{(t, x) \in[0,1] \times[0,1]}\left|\frac{\partial^{M+1}}{\partial t^{M+1}} u(t, x)\right|+\frac{1}{4} \max _{(t, x) \in[0,1] \times[0,1]}\left|\frac{\partial^{M+1}}{\partial x^{M+1}} u(t, x)\right|\right.} \\
& \left.+\frac{1}{16} \max _{(t, x) \in[0,1] \times[0,1]}\left|\frac{\partial^{2 M+2} u(t, x)}{\partial t^{M+1} \partial x^{M+1}}\right| \frac{1}{M^{M+1}}\right] \frac{1}{M^{M+1}} .
\end{aligned}
$$

\section{Operational matrices of integrations and differentiations}

The concerned section is committed to the study of operational matrices of fractional order derivatives and integrals based on Shifted Legendre polynomials, which are needed throughout in this paper. The operational matrices of fractional order derivatives and integration by Legendre polynomials in case of single variable are studied in [20]. In this article we develop the operational matrices of fractional order integration and differentiations and extend the notation to case of two variables.

Theorem 3.1. Consider $\Psi_{M^{2}}(t, x)$ as given in (2.3), then the $p$ order integration of $\Psi_{M^{2}}(t, x)$ w.r.t " $t$ " is given by

$$
\mathcal{J}^{p} \Psi_{M^{2}}(t, x) \simeq \hat{S}_{M^{2} \times M^{2}}^{(p, t)} \Psi_{M^{2}}(t, x)
$$

with $\hat{\mathrm{S}}_{\mathrm{M}^{2} \times \mathrm{M}^{2}}^{(\mathrm{p}, \mathrm{being}}$ the operational matrix of integration of order $\mathrm{p}>0$ given by 


$$
\hat{\mathrm{S}}_{\mathrm{M}^{2} \times M^{2}}^{(p, t)}=\left[\begin{array}{cccccc}
\Lambda_{1,1, k} & \Lambda_{1,2, k} & \cdots & \Lambda_{1, r, k} & \cdots & \Lambda_{1, M^{2}, k} \\
\Lambda_{2,1, k} & \Lambda_{2,2, k} & \cdots & \Lambda_{2, r, k} & \cdots & \Lambda_{2, M^{2}, k} \\
\vdots & \vdots & \vdots & \vdots & \vdots & \vdots \\
\Lambda_{\mu, 1, k} & \Lambda_{\mu, 2, k} & \cdots & \Lambda_{\mu, r, k} & \cdots & \Lambda_{\mu, M^{2}, k} \\
\vdots & \vdots & \vdots & \vdots & \vdots & \vdots \\
\Lambda_{M^{2}, 1, k} & \Lambda_{M^{2}, 2, k} & \cdots & \Lambda_{M^{2}, r, k} & \cdots & \Lambda_{M^{2}, M^{2}, k}
\end{array}\right],
$$

and $r=M i+j+1, \mu=M a+b+1, \Lambda_{\mu, r, k}=\mathbb{E}_{i, j, a, b, k}$ for $i, j, a, b=0,1,2, \ldots, m$,

$$
\mathbb{E}_{i, j, a, b, k}=\sum_{k=0}^{a} \delta_{i, b}(2 i+1) \sum_{l=0}^{i} \frac{(-1)^{i+l+a+k}(i+l) !(a+k) !}{(i-l) !(l !)^{2}(k+p+l+1)(a-k) ! k ! \Gamma(p+k+1)} .
$$

Proof. To prove the above result, applying the fractional integral of order $p$ on (2.1), w.r.t " $t$," we get

$$
\mathcal{J}^{p} \mathrm{~L}_{n}(\mathrm{t}, \mathrm{x})=\mathcal{J}^{p} \mathrm{~L}_{\mathrm{a}}(\mathrm{t}) \mathrm{L}_{\mathrm{b}}(\mathrm{x})=\sum_{\mathrm{k}=0}^{\mathrm{a}} \frac{(-1)^{\mathrm{a}+\mathrm{k}}(\mathrm{a}+\mathrm{k}) !}{(\mathrm{a}-\mathrm{k}) !(\mathrm{k} !)^{2}} \mathrm{I}_{\mathrm{t}}^{\mathrm{p}} \mathrm{t}^{\mathrm{k}} \mathrm{L}_{\mathrm{b}}(\mathrm{x}) .
$$

In view of definition of fractional derivative, equation (3.1), can be written as

$$
\mathcal{J}^{p} \mathrm{~L}_{\mathrm{a}}(\mathrm{t}) \mathrm{L}_{\mathrm{b}}(\mathrm{x})=\sum_{\mathrm{k}=0}^{\mathrm{i}} \frac{(-1)^{\mathrm{a}+\mathrm{k}}(\mathrm{a}+\mathrm{k}) !}{(\mathrm{a}-\mathrm{k}) !(\mathrm{k} !) \Gamma(k+p+1)} \mathrm{t}^{\mathrm{k}+\mathrm{p}} \mathrm{L}_{\mathrm{b}}(\mathrm{x}), \quad \mathrm{b}=1,2,3, \ldots, \mathrm{M} .
$$

Now, approximating $t^{k+p} L_{b}(x)$ by Legendre polynomial in two variables, we get

$$
\mathrm{L}_{\mathrm{b}}(\mathrm{x}) \mathrm{t}^{\mathrm{k}+\mathrm{p}} \approx \sum_{i=0}^{\mathrm{m}} \sum_{j=0}^{\mathrm{m}} \mathbb{E}_{i j} \mathrm{~L}_{i}(\mathrm{t}) \mathrm{L}_{j}(\mathrm{x}),
$$

where $\mathbb{E}_{i j}=(2 i+1)(2 j+1) \int_{0}^{1} \int_{0}^{1} L_{b}(x) t^{k+p} L_{i}(t) L_{j}(x) d t d x$, and using the orthogonality relation, we get

$$
\mathbb{E}_{i j}= \begin{cases}0, & \text { if } b \neq j, \\ (2 i+1) \sum_{l=0}^{i} \frac{(-1)^{i+j}(i+1) !}{(i-l) !(l !)^{2}(k+l+p+1)}, & \text { if } b=j .\end{cases}
$$

We can rewrite the above equation (3.3) as

$$
\mathbb{E}_{i j, b}=\delta_{j, b}(2 i+1) \sum_{l=0}^{i} \frac{(-1)^{i+j}(i+1) !}{(i-l) !(l !)^{2}(k+l+p+1)},
$$

where $\delta_{j, b}=\left\{\begin{array}{ll}0, & \text { if } b \neq j, \\ 1, & \text { if } b=j .\end{array}\right.$ Now, substitution the values in equation (3.2), we obtain

$$
\begin{aligned}
\mathcal{J P}^{p} \mathrm{~L}_{a}(\mathrm{t}) \mathrm{L}_{b}(x) & =\sum_{k=0}^{a} \frac{(-1)^{a+k}(a+k) !}{(a-k) !(k !) \Gamma(k+p+1)} \sum_{i=0}^{m} \sum_{j=0}^{m} \mathbb{E}_{i j, b} L_{i}(t) L_{j}(x), \\
& =\sum_{i=0}^{m} \sum_{j=0}^{m} \sum_{k=0}^{a} \frac{(-1)^{a+k}(a+k) !}{(a-k) !(k !) \Gamma(k+p+1)} \mathbb{E}_{i j, b} L_{i}(t) L_{j}(x), \\
& =\sum_{i=0}^{m} \sum_{j=0}^{m} \mathbb{E}_{i j, b, a, k} L_{i}(t) L_{j}(x),
\end{aligned}
$$

where

$$
\mathbb{E}_{i, j, a, b, k}=\sum_{k=0}^{a} \delta_{i, b}(2 i+1) \sum_{l=0}^{i} \frac{(-1)^{i+l+a+k}(i+l) !(a+k) !}{(i-l) !(l !)^{2}(k+p+l+1)(a-k) ! k ! \Gamma(p+k+1)}
$$


Using the notation like

$$
r=M i+j+1, \mu=M a+b+1, \Lambda_{\mu, r, k}=\mathbb{E}_{i, j, a, b, k} \text { for } i, j, a, b=0,1,2, \ldots, m,
$$

we get

$$
\mathcal{J}^{p} L_{a}(t) L_{b}(x)=\sum_{\mu=1}^{M^{2}} \sum_{r=1}^{M^{2}} \Lambda_{\mu, r, k} L_{i}(t) L_{j}(x), \text { for } a, b, i, j=0,1,2, \ldots, m .
$$

Consequently, the desired result follows.

Theorem 3.2. Consider the function vector $\Psi_{M^{2}}(t, x)$ defined in (2.3), then the fractional order differentiation of $\Psi_{M^{2}}(t, x)$ w.r.t " $x$ " is given by

$$
{ }^{c} \mathcal{D}^{p}\left(\Psi_{M^{2}}(t, x)\right) \simeq \mathbb{H}_{M^{2} \times M^{2}}^{(p, x)} \Psi_{M^{2}}(t, x),
$$

where $\mathbb{H}_{M^{2} \times M^{2}}^{(p, x)}$ is the operational matrix given as

$$
\mathbb{H}_{M^{2} \times M^{2}}^{(p, x)}=\left[\begin{array}{cccccc}
\coprod_{1,1, k} & \coprod_{1,2, k} & \cdots & \coprod_{1, r, k} & \cdots & \coprod_{1, M^{2}, k} \\
\coprod_{2,1, k} & \coprod_{2,2, k} & \cdots & \coprod_{2, v r, k} & \cdots & \coprod_{2, M^{2}, k} \\
\vdots & \vdots & \vdots & \vdots & \vdots & \vdots \\
\coprod_{\mu, 1, k} & \coprod_{\mu, 2, k} & \cdots & \coprod_{\mu, r, k} & \cdots & \coprod_{\mu, M^{2}, k} \\
\vdots & \vdots & \vdots & \vdots & \vdots & \vdots \\
\coprod_{M^{2}, 1, k} & \coprod_{M^{2}, 2, k} & \cdots & \coprod_{M^{2}, \mu, k} & \cdots & \coprod_{M^{2}, M^{2}, k}
\end{array}\right],
$$

and $\mu=\mathrm{Ni}+\mathrm{j}+1, \mathrm{r}=\mathrm{Na}+\mathrm{b}+1$, for $i, j, a, b=0,1,2, \ldots, n$,

$$
\coprod_{\mu, r, k}=E_{i, j, b, a, k}=\sum_{k=\lceil p\rceil}^{a} \delta_{i, a}(2 j+1) \sum_{l=0}^{j} \frac{(-1)^{j+l+b+k}(j+l) !(b+k) !}{(j-l) !(l !)^{2}(k+l-p+1)(b-k) ! \Gamma(k-p+1)}
$$

with

$$
\mathrm{E}_{i, j, b, a, k}=0 \text {, if } b<p .
$$

Similarly, the fractional order differentiation of $\Psi_{\mathrm{M}^{2}}(\mathrm{t}, \mathrm{x})$ w.r.t " $\mathrm{t}$ " is given as

$$
{ }^{c} \mathcal{D}^{p}\left(\Psi_{M^{2}}(t, x)\right) \simeq \mathbb{H}_{M^{2} \times M^{2}}^{(p, t)} \Psi_{M^{2}}(t, x),
$$

where $\mathbb{H}_{\mathrm{M}^{2} \times \mathrm{M}^{2}}^{(\mathrm{p}, \mathrm{t})}$ is the operational matrix given as

$$
\mathbb{H}_{M^{2} \times M^{2}}^{(p, t)}=\left[\begin{array}{cccccc}
\coprod_{1,1, k} & \coprod_{1,2, k} & \cdots & \coprod_{1, r, k} & \cdots & \coprod_{1, M^{2}, k} \\
\coprod_{2,1, k} & \coprod_{2,2, k} & \cdots & \coprod_{2, r, k} & \cdots & \coprod_{2, M^{2}, k} \\
\vdots & \vdots & \vdots & \vdots & \vdots & \vdots \\
\coprod_{\mu, 1, k} & \coprod_{\mu, 2, k} & \cdots & \coprod_{\mu, r, k} & \cdots & \coprod_{\mu, M^{2}, k} \\
\vdots & \vdots & \vdots & \vdots & \vdots & \vdots \\
\coprod_{M^{2}, 1, k} & \coprod_{M^{2}, 2, k} & \cdots & \coprod_{M^{2}, \mu, k} & \cdots & \coprod_{M^{2}, M^{2}, k}
\end{array}\right]
$$

and $\mu=N i+j+1, r=N a+b+1$, for $i, j, a, b=0,1,2, \ldots, n$,

$$
\coprod_{\mu, r, k}=E_{i, j, b, a, k}=\sum_{k=\lceil p\rceil}^{a} \delta_{i, a}(2 j+1) \sum_{l=0}^{j} \frac{(-1)^{j+l+b+k}(j+l) !(b+k) !}{(j-l) !(l !)^{2}(k+l-p+1)(b-k) ! \Gamma(k-p+1)}
$$

with

$$
E_{i, j, b, a, k}=0 \text {, if } b<p .
$$

Proof. Like the proof of Theorem 3.1, one can easily obtain the proof. 


\section{Numerical scheme based on the operational matrices}

The present section is devoted to used the aforesaid operational matrices to perform numerical analysis of the proposed coupled system of FPDEs given as

$$
\begin{aligned}
& { }^{c} D_{t}^{p} u(t, x)+{ }^{c} D_{x}^{q} v(t, x)=f(t, x), t, x \in[0,1], \\
& { }^{c} D_{t}^{p} v(t, x)+{ }^{c} D_{x}^{q} u(t, x)=g(t, x), t, x \in[0,1],
\end{aligned}
$$

subject to the initial conditions

$$
u(0, x)=\phi(x), v(0, x)=\theta(x)
$$

First of all, we convert the system of coupled equations (4.1) into the simple algebraic equations, which are very easy to soluble.

We approximate the partial fractional order derivatives by Legendre polynomial of order $M$ in two variables as

$$
{ }^{c} D_{t}^{p} u(t, x)=K_{M^{2}} \Psi_{M^{2}}(t, x), \quad{ }^{c} D_{t}^{q} v(t, x)=\mathbb{Z}_{M^{2}} \Psi_{M^{2}}(t, x) .
$$

Now, applying integral of order $p, q$, respectively, with respect to $t$ and inview of Definition 2.1, we have

$$
u(t, x)=c_{0}+\mathbb{K}_{M^{2}} \hat{S}_{M^{2} \times M^{2}}^{(p, t)} \Psi_{M^{2}}(t, x), v(t, x)=c_{1}+\mathbb{Z}_{M^{2}} \hat{S}_{M^{2} \times M^{2}}^{(q, t)} \Psi_{M^{2}}(t, x) .
$$

Using the initial conditions of (4.2) yields that

$$
u(t, x)=\phi(x)+\mathbb{K}_{M^{2}} \hat{S}_{M^{2} \times M^{2}}^{(p, t)} \Psi_{M^{2}}(t, x), \quad v(t, x)=\theta(x)+\mathbb{Z}_{M^{2}} \hat{S}_{M^{2} \times M^{2}}^{(q, t)} \Psi_{M^{2}}(t, x) .
$$

Now, we approximat $\phi(x)$ and $\theta(x)$ as follows

$$
\phi(x)=\mathbb{F}^{(1)} \Psi_{M^{2}}(t, x), \quad \theta(x)=\mathbb{F}^{(2)} \Psi_{M^{2}}(t, x) .
$$

Now inview of the relation obtained in (4.4), we have

$$
u(t, x)=\mathbb{F}^{(1)} \Psi_{M^{2}}+\mathbb{K}_{M^{2}} \hat{S}_{M^{2} \times M^{2}}^{(p, t)} \Psi_{M^{2}}(t, x), v(t, x)=\mathbb{F}^{(2)} \Psi_{M^{2}}+\mathbb{Z}_{M^{2}} \hat{S}_{M^{2} \times M^{2}}^{(q, t)} \Psi_{M^{2}}(t, x) .
$$

Applying fractional derivatives of order $p$ and $q$ on equations (4.5), one can gets

$$
\begin{aligned}
& { }^{c} D_{t}^{p} u(t, x)=\mathbb{F}^{(1)} \mathbb{H}_{M^{2} \times M^{2}}^{(p, t)} \Psi_{M^{2}}(t, x)+\mathbb{K}_{M^{2}} \hat{S}_{M^{2} \times M^{2}}^{(p, t)} \mathbb{H}_{M^{2} \times M^{2}}^{(p, t)} \Psi_{M^{2}}(t, x), \\
& { }^{c} D_{t}^{q} v(t, x)=\mathbb{F}^{(2)} H_{M^{2} \times M^{2}}^{(q, t)} \Psi_{M^{2}}(t, x)+\mathbb{Z}_{M^{2}} \hat{S}_{M^{2} \times M^{2}}^{(q, t)} H_{M^{2} \times M^{2}}^{(p, t)} \Psi_{M^{2}}(t, x) .
\end{aligned}
$$

Similarly, we find the relation for ${ }^{c} D_{\chi}^{q} u(t, x)$ and ${ }^{c} D_{x}^{p} v(t, x)$ as follows:

$$
\begin{aligned}
{ }^{c} D_{\chi}^{q} u(t, x) & =\mathbb{F}^{(1)} H_{M^{2} \times M^{2}}^{(q, x)} \Psi_{M^{2}}(t, x)+\mathbb{K}_{M^{2}} \hat{S}_{M^{2} \times M^{2}}^{(q, x)} \mathbb{H}_{M^{2} \times M^{2}}^{(q, x)} \Psi_{M^{2}}(t, x), \\
{ }^{c} D_{x}^{p} v(t, x) & =\mathbb{F}^{(2)} H_{M^{2} \times M^{2}}^{(p, x)} \Psi_{M^{2}}(t, x)+Z_{M^{2}} \hat{S}_{M^{2} \times M^{2}}^{(p, x)} \mathbb{H}_{M^{2} \times M^{2}}^{(q, x)} \Psi_{M^{2}}(t, x) .
\end{aligned}
$$

Approximating $f(t, x)=G_{M^{2}}^{(1)} \Psi_{M^{2}}(t, x), g(t, x)=G_{M^{2}}^{(2)} \Psi_{M^{2}}(t, x)$ and using equations (4.3), (4.6), and (4.7) in our considered system of FPDEs (4.1), then we get as

$$
\begin{aligned}
& K_{M^{2}} \Psi_{M^{2}}(t, x)=-\mathbb{F}^{(2)} \mathbb{H}_{M^{2} \times M^{2}}^{(q, t)} \Psi_{M^{2}}(t, x)-\mathbb{Z}_{M^{2}} \hat{S}_{M^{2} \times M^{2}}^{(q, t)} \mathbb{H}_{M^{2} \times M^{2}}^{(p, t)} \Psi_{M^{2}}(t, x)+G_{M^{2}}^{(1)} \Psi_{M^{2}}(t, x), \\
& \mathbb{Z}_{M^{2}} \Psi_{M^{2}}(t, x)=-\mathbb{F}^{(1)} \mathbb{H}_{M^{2} \times M^{2}}^{(q, t)} \Psi_{M^{2}}(t, x)-\mathbb{K}_{M^{2}} \hat{S}_{M^{2} \times M^{2}}^{(q, t)} \mathbb{H}_{M^{2} \times M^{2}}^{(p, t)} \Psi_{M^{2}}(t, x)+G_{M^{2}}^{(2)} \Psi_{M^{2}}(t, x) .
\end{aligned}
$$


Now, we can write the above equation in matrix form as

$$
\begin{aligned}
{\left[\begin{array}{l}
\mathbb{K}_{M^{2}} u_{M^{2}}(t, x) \\
\mathbb{Z}_{M^{2}} u_{M^{2}}(t, x)
\end{array}\right]=} & {\left[\begin{array}{l}
-\mathbb{F}^{(2)} \mathbb{H}_{M^{2} \times M^{2}}^{(q, t)} \Psi_{M^{2}}(t, x) \\
-\mathbb{F}^{(1)} \mathbb{H}_{M^{2} \times M^{2}}^{(q, t)} \Psi_{M^{2}}(t, x)
\end{array}\right]+\left[\begin{array}{l}
-\mathbb{Z}_{M^{2}} \hat{S}_{M^{2} \times M^{2}}^{(q, t)} \mathbb{H}_{M^{2} \times M^{2}}^{(p, t)} \Psi_{M^{2}}(t, x) \\
-K_{M^{2}} \hat{S}_{M^{2} \times M^{2}}^{(q, t)} \mathbb{H}_{M^{2} \times M^{2}}^{(p, t)} \Psi_{M^{2}}(t, x)
\end{array}\right] } \\
& +\left[\begin{array}{l}
G_{M M^{2}}^{(1)} \Psi_{M^{2}}(t, x) \\
G_{M^{2}}^{(2)} \Psi_{M^{2}}(t, x)
\end{array}\right] .
\end{aligned}
$$

By taking transpose and rearranging the matrices of the system (4.8), we get

$$
\begin{aligned}
& {\left[\begin{array}{ll}
\mathbb{K}_{M^{2}}^{\top} & \mathbb{Z}_{M^{2}}^{\top}
\end{array}\right]\left[\begin{array}{ll}
\Psi_{M^{2}}(t, x) & O \\
O & \Psi_{M^{2}(t, x)}
\end{array}\right] } \\
&=-\left[\begin{array}{lll}
\mathbb{F}^{2} \mathbb{H}_{M^{2} \times M^{2}}^{(q, t)} & \mathbb{F}^{1} \mathbb{H}_{M^{2} \times M^{2}}^{(q, t)}
\end{array}\right]\left[\begin{array}{cc}
\Psi_{M^{2}}(t, x) & O \\
O & \Psi_{M^{2}(t, x)}
\end{array}\right] \\
&-\left[\begin{array}{lll}
\mathbb{Z}_{M^{2}}^{\top} \hat{S}_{M^{2} \times M^{2}}^{(q, t)} \mathbb{H}_{M^{2} \times M^{2}}^{(p, t)} & \mathbb{K}_{M^{2}}^{\top} \hat{S}_{M^{2} \times M^{2}}^{(q, t)} \mathbb{H}_{M^{2} \times M^{2}}^{(p, t)}
\end{array}\right]\left[\begin{array}{cc}
\Psi_{M^{2}}(t, x) & O \\
O & \Psi_{M^{2}}(t, x)
\end{array}\right] \\
&+\left[\begin{array}{lll}
G_{M^{2}}^{1} & G_{M^{2}}^{2}
\end{array}\right]\left[\begin{array}{cc}
\Psi_{M^{2}}(t, x) & O \\
O & \Psi_{M^{2}}(t, x)
\end{array}\right] .
\end{aligned}
$$

Let us denote

$$
A=\left[\begin{array}{ccc}
\Psi_{M^{2}}(t, x) & \mathbb{O} \\
O & \Psi_{M^{2}}(t, x)
\end{array}\right]
$$

Then equation (4.9), becomes

$$
\begin{aligned}
& {\left[\begin{array}{ll}
\mathbb{K}_{M^{2}}^{\top} & \mathbb{Z}_{M^{2}}^{\top}
\end{array}\right] A+\left[\begin{array}{ll}
\mathbb{F}^{2} \mathbb{H}_{M^{2} \times M^{2}}^{(q, t)} & \mathbb{F}^{1} \mathbb{H}_{M^{2} \times M^{2}}^{(q, t)}
\end{array}\right] A}
\end{aligned}
$$

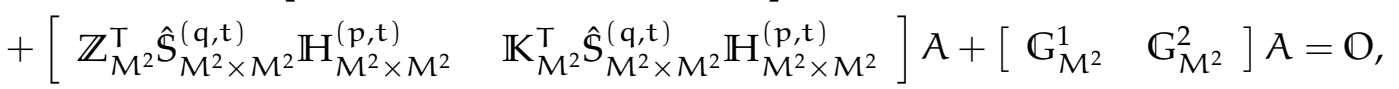

which implies that

$$
\begin{aligned}
& {\left[\begin{array}{ll}
\mathbb{K}_{M^{2}}^{\top} & \mathbb{Z}_{M^{2}}^{\top}
\end{array}\right]+\left[\begin{array}{ll}
\mathbb{F}^{2} \mathbb{H}_{M^{2} \times M^{2}}^{(q, t)} & \mathbb{F}^{1} \mathbb{H}_{M^{2} \times M^{2}}^{(q, t)}
\end{array}\right]}
\end{aligned}
$$

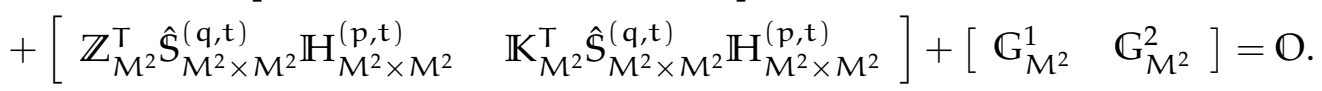

It is obvious that (4.10) is an algebraic equation of matrices, which can be solved for the unknown matrix

$$
\left[\begin{array}{ll}
\mathbb{K}_{M^{2}}^{\top} & \mathbb{Z}_{M^{2}}^{\top}
\end{array}\right] \text {. }
$$

After computation and putting its value in (4.5), one can get the approximate solution of the considered problem.

\section{Numerical examples}

The present section is concerning to test the above technique by some well known interest problems given bellow.

Example 5.1. Consider the coupled system of homogenous FPDEs given by

$$
\begin{aligned}
& { }^{c} D_{t}^{p} u(t, x)+{ }^{c} D_{x}^{q} v(t, x)=0,0<p, q \leqslant 1, t, x \in[0,1], \\
& { }^{c} D_{t}^{q} v(t, x)+{ }^{c} D_{x}^{q} u(t, x)=0,0<p, q \leqslant 1, t, x \in[0,1],
\end{aligned}
$$

subject to the initial conditions

$$
u(0, x)=\exp (x), v(0, x)=\exp (-x) .
$$

It should be noted that the exact solution of (5.1) at $p=q=1$ is given in [16] as 


$$
u(t, x)=\exp (x) \cosh (t)+\exp (-x) \sinh (t), v(t, x)=\exp (-x) \cosh (t)-\exp (x) \sinh (t)
$$

We find the approximate solutions using the proposed method and compared the results with the Legendre multiwavelets and Chebyshev multiwavelet of $u(t, x)$ and $v(t, x)$. For the afore said comparison we have taken scale level $M=6$ in Tables 1 and 2, respectively. We see that our proposed method is more efficient than the given method as shown in Tables 1 and 2 and Figures 1 and 2, respectively.

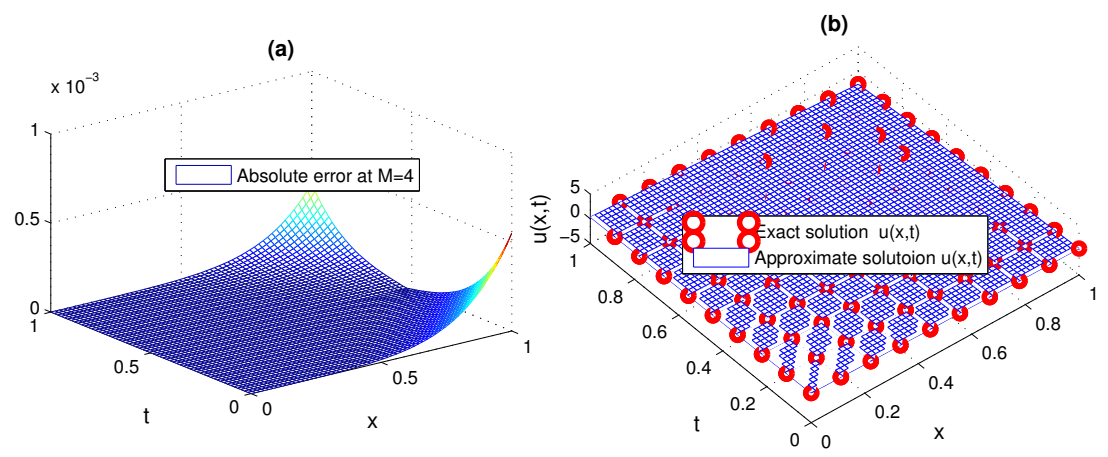

Figure 1: (a) Plot of absolute error for $u(t, x)$ at $M=4, p=q=0.9$. (b) Comparison of approximate and exact solution for $u(t, x)$ of Example 5.1 at $M=4$.

(c)
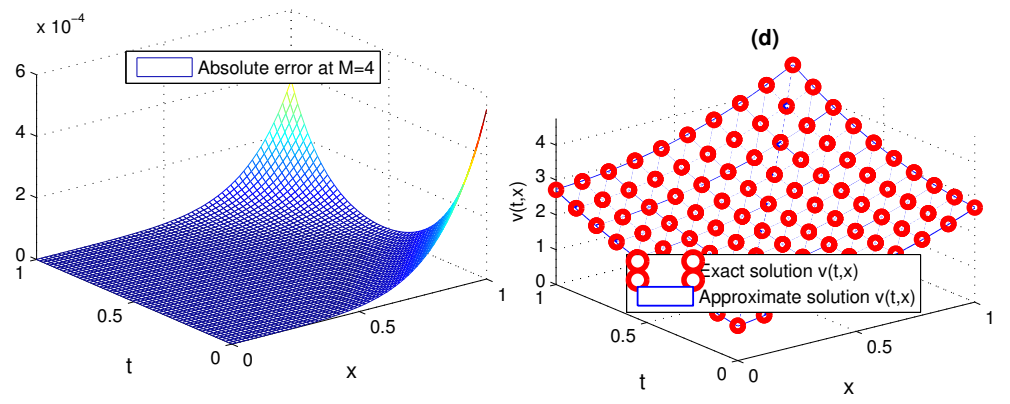

Figure 2: (c) Plot of absolute error for $v(t, x)$ at $M=4, p=q=0.9$. (d) Comparison of approximate and exact solution for $v(t, x)$ of Example 5.1 at $M=4$.

Table 1: Comparison between the results obtained by Legendre operational matrices method (LOM), Legendre Multiwavelet method (LMW), and Chebeshive multiwavelet method (CMW) of $u(t, x)$ for Example 5.1 at $p=q=1$.

\begin{tabular}{|c|c|c|c|}
\hline$x / t=0.1$ & $\left|u_{\text {Eaxct }}-u_{\mathrm{LOM}}\right|$ & $\left|\mathrm{u}_{\text {Eaxct }}-\mathrm{u}_{\mathrm{LMW}}\right|$ & $\left|\mathrm{u}_{\text {Eaxct }}-\mathrm{u}_{\mathrm{CMW}}\right|$ \\
\hline 0 & $1 \times 10^{-10}$ & $4.3 \times 10^{-5}$ & $4.3 \times 10^{-5}$ \\
0.1 & $1.9 \times 10^{-10}$ & $3.3 \times 10^{-5}$ & $3.3 \times 10^{-5}$ \\
0.2 & $4.3 \times 10^{-9}$ & $8.3 \times 10^{-6}$ & $8.3 \times 10^{-6}$ \\
0.3 & $2.6 \times 10^{-8}$ & $1.3 \times 10^{-5}$ & $1.3 \times 10^{-5}$ \\
0.4 & $1.8 \times 10^{-8}$ & $2.1 \times 10^{-5}$ & $2.1 \times 10^{-5}$ \\
0.5 & $8.4 \times 10^{-7}$ & $1.2 \times 10^{-5}$ & $1.2 \times 10^{-5}$ \\
0.6 & $9.7 \times 10^{-7}$ & $5.6 \times 10^{-6}$ & $5.6 \times 10^{-6}$ \\
0.7 & $3.8 \times 10^{-7}$ & $2.2 \times 10^{-5}$ & $2.2 \times 10^{-5}$ \\
0.8 & $6.5 \times 10^{-6}$ & $1.8 \times 10^{-5}$ & $1.8 \times 10^{-5}$ \\
0.9 & $5.4 \times 10^{-6}$ & $3.1 \times 10^{-5}$ & $3.1 \times 10^{-5}$ \\
1.0 & $7.8 \times 10^{-5}$ & $1.5 \times 10^{-4}$ & $4.3 \times 10^{-5}$ \\
\hline
\end{tabular}


Table 2: Comparison between the results obtained for $v(t, x)$ by Legendre operational matrices method (LOM), Legendre Multiwavelet method (LMW), and Chebyshev Multiwavelet method (CMW) for Example 5.1 at $p=q=1$.

\begin{tabular}{|c|c|c|c|}
\hline$x / \mathrm{t}=0.1$ & $\left|v_{\text {Eaxct }}-v_{\text {LOM }}\right|$ & $\left|v_{\text {Eaxct }}-u_{\text {LMW }}\right|$ & $\left|v_{\text {Eaxct }}-v_{\text {CMW }}\right|$ \\
\hline 0 & $1.2 \times 10^{-9}$ & $7.9 \times 10^{-5}$ & $7.9 \times 10^{-5}$ \\
0.1 & $1.0 \times 10^{-9}$ & $1.7 \times 10^{-5}$ & $1.7 \times 10^{-5}$ \\
0.2 & $3.9 \times 10^{-8}$ & $2.9 \times 10^{-5}$ & $2.9 \times 10^{-5}$ \\
0.3 & $2.0 \times 10^{-8}$ & $4.9 \times 10^{-6}$ & $4.9 \times 10^{-6}$ \\
0.4 & $1.4 \times 10^{-8}$ & $2.2 \times 10^{-5}$ & $2.2 \times 10^{-5}$ \\
0.5 & $7.8 \times 10^{-7}$ & $3.1 \times 10^{-5}$ & $3.1 \times 10^{-5}$ \\
0.6 & $4.9 \times 10^{-7}$ & $1.6 \times 10^{-5}$ & $1.6 \times 10^{-5}$ \\
0.7 & $6.5 \times 10^{-7}$ & $1.5 \times 10^{-5}$ & $1.5 \times 10^{-5}$ \\
0.8 & $1.2 \times 10^{-6}$ & $3.4 \times 10^{-5}$ & $3.4 \times 10^{-5}$ \\
0.9 & $2.9 \times 10^{-6}$ & $5.0 \times 10^{-6}$ & $4.9 \times 10^{-6}$ \\
1.0 & $1.0 \times 10^{-5}$ & $1.7 \times 10^{-4}$ & $7.9 \times 10^{-5}$ \\
\hline
\end{tabular}

Example 5.2. Consider the coupled system of homogeneous FPDEs given by

$$
\begin{aligned}
& { }^{c} D_{t}^{0.9} u(t, x)+{ }^{c} D_{x}^{0.8} v(t, x)=f(t, x), t, x \in[0,1], \\
& { }^{c} D_{t}^{0.9} v(t, x)+{ }^{c} D_{x}^{0.8} u(t, x)=g(t, x), t, x \in[0,1],
\end{aligned}
$$

subject to the initial conditions

$$
u(0, x)=x^{2}+1, v(0, x)=\exp (x)
$$

where

$$
f(t, x)=\sum_{k=0}^{\infty} \frac{(-1)^{k} t^{k-0.9}}{\Gamma(k-0.1)}+\sum_{k=0}^{\infty} \frac{x^{k-0.8}}{\Gamma(k-0.2)}, g(t, x)=\frac{t^{1.1}}{\Gamma(2.1)}+\frac{x^{1.1}}{\Gamma(2.1)} .
$$

It is to be noted that the exact solution of (5.2) at $p=q=1$ is given as

$$
u(t, x)=x^{2}+\exp (-t) v(t, x)=t^{2}+\exp (x) .
$$

We find the approximate solutions using the proposed method of $u(t, x)$ and $v(t, x)$. We see that our proposed method is more efficient and provide more excellent solutions even at a small scale level as shown in Figures 3 and 4, respectively. Further we also computed the absolute error at various scale levels in Table 3. We see that as scale level is increasing, the absolute error is decreasing and vice versa, which has been shown in Table 3 .

(a)

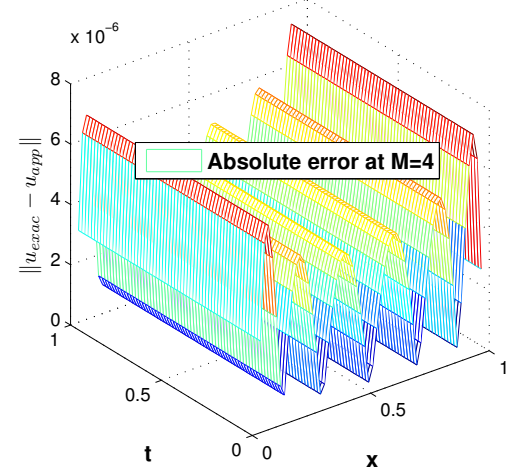

(b)

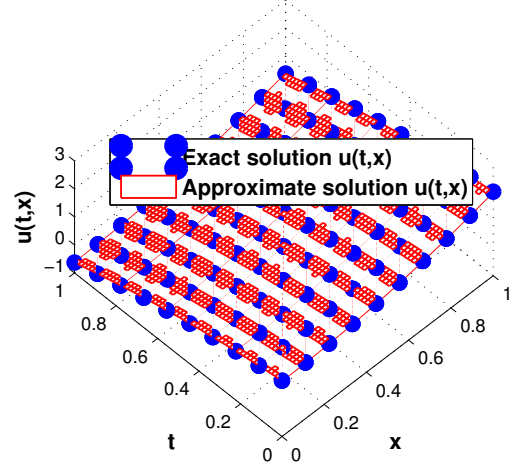

Figure 3: (a) Plot of absolute error for $u(t, x)$ at $M=4$. (b) Comparison of approximate and exact solution for $u(t, x)$ of Example 5.2 at $M=4$. 


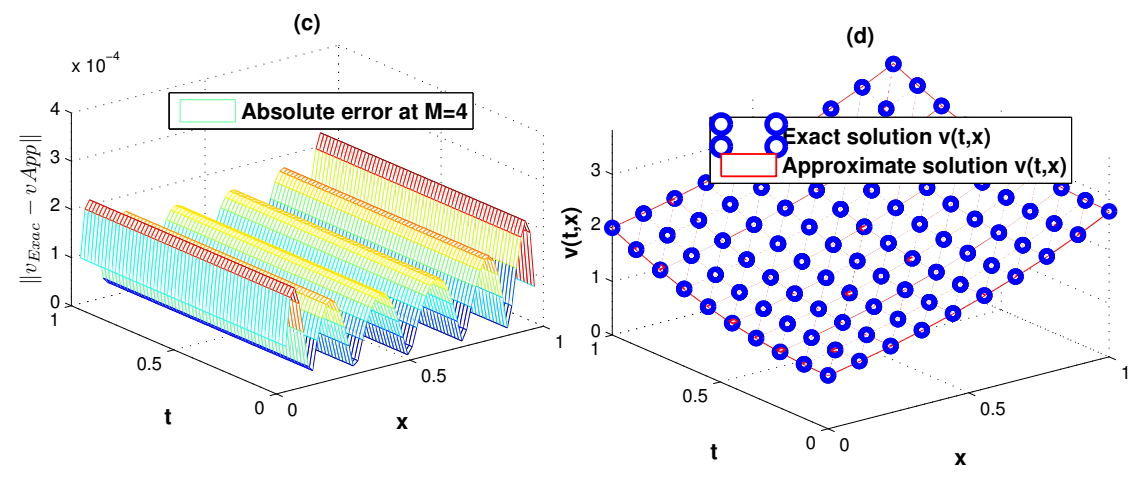

Figure 4: (c) Plot of absolute error for $v(t, x)$ at $M=4$. (d) Comparison of approximate and exact solution for $v(t, x)$ of Example 5.2 at $M=4$.

Table 3: Absolute error at different scale levels in the solutions recited by Legendre operational matrices method for Example 5.2 using fractional order at $\mathrm{p}=0.9, \mathrm{q}=0.8$.

\begin{tabular}{|c|c|c|c|}
\hline$(t, x)$ & Scale $=M$ & $\left|\mathfrak{u}_{\text {Exact }}-\mathfrak{u}_{\text {App }}\right|$ & $\left|v_{\text {Exact }}-v_{\text {App }}\right|$ \\
\hline$(0.1,0.1)$ & 4 & $8.17634 \times 10^{-6}$ & $2.00033 \times 10^{-4}$ \\
$(0.1,0.3)$ & 5 & $2.09597 \times 10^{-7}$ & $1.09597 \times 10^{-4}$ \\
$(0.4,0.5)$ & 6 & $1.29274 \times 10^{-8}$ & $4.9278 \times 10^{-5}$ \\
$(0.5,0.5)$ & 7 & $1.00725 \times 10^{-8}$ & $1.00074 \times 10^{-5}$ \\
$(0.6,0.4)$ & 8 & $1.55176 \times 10^{-10}$ & $2.8921 \times 10^{-6}$ \\
$(0.4,0.6)$ & 10 & $1.55176 \times 10^{-10}$ & $1.55176 \times 10^{-8}$ \\
$(0.7,0.2)$ & 12 & $3.01549 \times 10^{-11}$ & $1.00345 \times 10^{-8}$ \\
$(0.3,0.7)$ & 14 & $9.05935 \times 10^{-12}$ & $6.55176 \times 10^{-10}$ \\
$(0.8,0.1)$ & 15 & $1.51204 \times 10^{-13}$ & $2.12346 \times 10^{-12}$ \\
$(0.9,0.2)$ & 16 & $2.93874 \times 10^{-14}$ & $7.36153 \times 10^{-13}$ \\
$(0.4,0.9)$ & 18 & $8.82871 \times 10^{-16}$ & $9.5016 \times 10^{-15}$ \\
$(1.0,1.0)$ & 20 & $1.47354 \times 10^{-16}$ & $8.23160 \times 10^{-16}$ \\
\hline
\end{tabular}

\section{Conclusion}

In the aforesaid analysis, we have successfully obtained a numerical scheme for the approximate solutions of coupled system of FPDEs subject to initial conditions. The results obtained by the proposed method have been compared with the results received by using Legendre and Chebyshev Multiwavelet method [16] for Example 5.1. From the comparison, one can easily understood that the proposed method is an efficient and reliable as compared to the mentioned methods. In the mentioned methods discretization is used while in the proposed method it has of no need. The method is computer oriented.

\section{Acknowledgment}

We are really thankful to the reviewers for their useful suggestions. This work was supported by the National Natural Science Foundation of China (11571378).

\section{References}

[1] R. T. Baillie, Long memory processes and fractional integration in econometrics, J. Econometrics, 73 (1996), 5-59. 1

[2] M. Caputo, Linear modles of dissipation whose Q is almost frequenty independent-II, J. R. Astr. Soc., 13 (1967), 529-539. 1

[3] S. Das, Functional Fractional Calculus for System Identification and Controls, Springer, Berlin, (2008). 1

[4] M. Deghan, S. A. Yousefi, A. Lotfi, The use of He's variational iteration method for solving the telegraph and fractional telegraph equations, Int. J. Numer. Methods Biomed. Eng., 27 (2011), 219-231. 1 
[5] E. H. Doha, A. H. Bhrawy, R. M. Hafez, On shifted Jacobi spectral method for high-order multi-point boundary value problems, Commun. Nonlinear Sci. Numer. Simul., 17 (2012), 3802-3810. 2.4

[6] M. Gasea, T. Sauer, On the history of multivariate polynomial interpolation, J. Comput. Appl. Math., 122 (2000), 23-35. 2.4

[7] Y. Z. Hu, Y. Luo, Z. Y. Lu, Analytical solution of the linear fractional differential equation by Adomian decomposition method, J. Comput. Appl. Math., 215 (2008), 220-229. 1

[8] R. A. Khan, H. Khalil, A new method based on legendre polynomials for solution of system of fractional order partial differential equations, Int. J. Comput. Math., 91 (2014), 2554-2567. 1, 1

[9] A. A. Kilbas, H. M. Srivastava, J. J. Trujillo, Theory and Applications of Fractional Differential Equations, Elsevier Science B.V., Amsterdam, (2006). 1

[10] D. Kumar, J. Singh, S. Kumar, Numerical computation of fractional multi-dimensional diffusion equations by using a modified homotopy perturbation method, J. Asso. Arab Universities Basic Appl. Sci., 17 (2015), 20-26. 1

[11] Y. L. Li, Solving a nonlinear fractional differential equation using Chebyshev wavelets, Commun. Nonlinear Sci. Numer. Simul., 15 (2010), 2284-2292. 1

[12] R. L. Magin, Fractional calculus in bioengineering-part 1, Crit. Rev. Biomed. Eng., 32 (2004), 104 pages. 1

[13] R. L. Magin, Fractional calculus in bioengineering-part 2, Crit. Rev. Biomed. Eng., 32 (2004), 90 pages.

[14] R. L. Magin, Fractional calculus in bioengineering-part 3, Crit. Rev. Biomed. Eng., 32 (2004), 183 pages.

[15] F. Mainardi, Fractals and Fractional Calculus Continuum Mechanics, Springer-Verlag, Vienna, (1997). 1

[16] M. A. Mohamed, M. S. Torky, Solution of Linear System of Partial Differential Equations by Legendre Multiwavelet and chebyshev multiwavelet, Int. J. Sci. Inno. Math. Research, 2 (2014), 966-976. 5.1, 6

[17] Z. Odibat, S. Momani, A generalized differential transform method for linear partial differential equations of fractional order, Appl. Math. Lett., 21 (2008), 194-199. 1

[18] I. Podlubny, Fractional Differential Equations, Acedemic Press, San Diego, (1999). 1, 2.1, $2.2,2.3$

[19] D. Rostamy, K. Karimi, E. Mohamadi, Solving fractional partial differential equations by an efficient new basis, Int. J. Appl. Math. Comput., 5 (2013), 6-21. 1

[20] A. Saadatmandi, M. Deghan, A new operational matrix for solving fractional-order differential equation, Comput. Math. Appl., 59 (2010), 1326-1336. 2.1, 3

[21] V. E. Tarasov, Fractional Integro Differentional Equations For Electromagnetic Waves in Dielectric Media, Theo. Math. Phy., 158 (2009), 355-359. 1

[22] Y. Q. Yang, Y. P. Ma, L. F. Wang, Legendre polynomials operational matrix method for solving fractional partial differential equations with variable coefficients, Math. Prob. Eng., 2015 (2015), 9 pages. 1, 2.4

[23] S. P. Yang, A. Xiao, H. Su, Convergence of the variational iteration method for solving multi-order fractional differential equations, Comput. Math. Appl., 60 (2010), 2871-2879. 1 